\title{
CURSORY REMARKS
}

\section{ON \\ S M A L L - P O X,}

AS IT OCCURS

\section{SUBSEQUENT TO VACCINATION.}

By GEORGE GREGORY, M. D.

rHYSICIAN TO THE hospital FOR SMALI-POX AND VACCiNation, AT ST. Pancras.

\section{Read Jan. 7, 1823.}

THE acknowledged frequency of cases of smallpox subsequent to vaccination, in all parts of the country, is such as to have excited, in no inconsiderable degree, the fears of many, and the anxieties of all. No one can look back upon the history of the last few years without feeling sensible that these unpleasant occurrences are on the increase, and it becomes, therefore, a matter, not of curiosity merely, but of real necessity, to attempt some regular investigation of the subject. The difficulty cannot be met by mere reference to the fact, that small-pox, once gone through, does not always secure to the subject immunity from a second attack. Cases of small-pox after vaccination are, beyond all comparison, more frequent than cases of secondary small-pox. The latter 
were, at all times, objects of curiosity, even to the older members of the profession. There are few. who have not seen repeated instances of the former. Within the walls of the Small-pox Hospital, the latter were rarely seen. The former constitute, at the present time, a considerable proportion of the admissions into that institution.

With the view of illustrating this point, and also of showing how far the prevalence of small-pox, after vaccination, is on the increase, $I$ have, in Table No. 1, given the total number of admissions into the Small-pox Hospital in ten different years, distinguishing such as occurred after real or presumed vaccination*. From this table it appears, that, in the year 1810, the proportion of cases of small-pox succeeding vaccination to the whole number of admissions, was as 1 in 80 ; in 1815, as 1 in 17; in 1819, as 1 in 6 ; in 1821, as 1 in 4 ; and during the year 1822, as 1 in $3 \frac{1}{2}$.

Above 100 cases have occurred at the Smallpox Hospital during the last three years, the greater number of which fell under my own observation. Fifty-seven were admitted in 1822. The opportunities which have thus been afforded to me

* All cases are here entered as having undergone vaccination, where the cicatrices were apparent, or failing that criterion, where the patient had a distinct recollection of the arm having risen, and of the general progress of the disease. Such cases as were known to have failed are excluded. 
of observing small-pox, as it occurs subsequent to vaccination, have been tolerably extensive, and sanction, in some degree, the liberty which I presume to take, of laying the results of my observation before the notice of my professional brethren.

The occurrence of small-pox after vaccination is a subject of very considerable pathological interest. Independent of its importance, as a question affecting the world at large, it affords curious matter of enquiry to the investigator of disease. It forms a link in that chain of facts which bear upon the general influence of the variolous poison upon the animal economy, and the several modifications of which it is susceptible; and, to be thoroughly understood, it must be viewed in conjunction with them. The subject is one of no ordinary difficulty; but it is not my intention, on the present occasion, to enter upon it in that detail which I believe it to deserve. My remarks will be very cursory, and my principal design to put upon the records of the Society some evidence that no fact which has reference to the great question of vaccine protection, is overlooked of concealed.

I am thoroughly sensible of the extreme delicacy of this enquiry. The mere agitation of the question in a Society like this may be deprecated by some as altogether unwarranted and uncalled for ; and, from the distrust of vaccination which it 
seems to imply, calculated to occasion much serious evil. Were I not satisfied, that this view of the subject is overstrained, I would not proceed. It is, however, clear to me, that vaccination is now so well established, that no real danger can arise from examining, even in the strictest manner, every phenomenon connected with it. A large proportion of mothers, in the present day, were themselves vaccinated, and, therefore, the popular prejudices may now be considered as in favour of vaccination rather than against it. So far from anticipating evil, I look forward to the public good being benefited by the free discussion of the subject. Many persons have been brooding in secret over the failures of vaccination, and appear to have a fear of expressing their sentiments concerning it, or of meeting the question, in any way, openly. To them the avowed investigation of the subject will, I am persuaded, prove satisfactory. But, besides this, it is only by candid discussion that we shall ever be able to determine that highly important point, how far the failures of vaccination are owing to causes under our control; and how far, therefore, there exists a reasonable probability of obviating them, either wholly or partially, so as to increase the security of the vaccinated.

I shall first enquire in what manner, and to what extent, the effects of the variolous poison upon the animal economy are modified by the infinence, previously exerted, of the vaccine virus. 
I shall then offer a few reflections on the causes of the occurrence of small-pox after vaccination, and on the sources of difference in the degree of modifying influence which vaccination exerts.

It is almost unnecessary to remark, in the first place, that, in a very large proportion of cases, the same immunity is afforded by vaccination as by once undergoing the genuine variolous disease. What the exact proportion is, we are unable to ascertain. It does not even appear that any approximation to the truth, which can be much relied on, has yet been made. We may even go further, and be warranted in saying, that no calculations tending to establish this point, which are made in the present day, can reasonably be expected to hold good in future; and the reason is obvious. The failures of vaccination are now far more numerous than they were ten years ago, and no certainty exists that they have yet reached their maximum.

In cases where the vaccine virus fails to impart a perfect security from the future influence of the variolous poison, it serves, at least, to modify certain of its effects. These it is important to investigate.

1. Vaccination does not appear to lessen the violence, or shorten the duration, of the first or eruptive stage of fever, which is generally as se- 
vere, and even sometimes severer and longer in its duration than that of the casual confluent small-pox.

2. It does not appear in like manner to influence the quantity of eruption upon the skin, so much, at least, as has been generally imagined. It is true that, in many cases of small-pox, subsequent to vaccination, the eruption has been very scanty; but, in a large number also, I have seen it very copious, more particularly about the face, breast, and upper extremities, and occasionally fully equal, in point of quantity, to what is seen in the worst kinds of confluent or coherent natural small-pox.

3. The great power of vaccination unquestionably consists in modifying the progress of inflammation in the variolous eruption; and here it cannot fail to attract observation, how strikingly opposed to each other, in this respect, are the influences of inoculation and of vaccination. Inoculation lessens the quantity of eruption, but does not alter, in the slightest degree, the progress of inflammation in that which is brought out. . Vaccination, on the other hand, while it does not sensibly affect the quantity of eruption, always influences, more or less, the progress of inflammation, however copious the eruption may be. The same desirable result, the diminution of mortality, is obtained in either way. By checking the quan- 
tity of eruption, or the degree to which inflammation in it extends, the disease is prevented from bringing on those impediments to the functions of respiration and perspiration, which occasion secondary fever, and endanger life.

In all, or nearly all cases of natural and inoculated small-pox, the eruption proceeds to ulceration, more or less superficial, according to the violence of the disease; and the ulcers heal by the common process of scabbing and cicatrization. In cases of small-pox, however, subsequent to vaccination, the cutaneous inflammation is checked at so early a period, that the fluid in the vesicles seldom reaches the state of pus, the cutis vera is never ulcerated, and consequently the healing process takes place by the conversion of the vesicles into tubercles, and their subsequent desquamation. This constitutes a very well-marked and important character of the vaccine or modified small-pox. A similar modification of the variolous inflammation of the fauces and trachea undoubtedly takes place; but the exact nature of the difference it is, in this case, more difficult to define.

4. Though vaccination modifies, in a large proportion of cases, the progress of inflammation in the skin and throat, it is curious to observe that it does not always affect the course of the disease, when the variolous poison fixes itself on other 
parts, more particularly on the brain*. It is, in this manner, that small-pox, after vaccination, occasionally proves fatal.

The following cases will illustrate the position which is here advanced.

\section{CASE $I$.}

Variola succeeding vaccination, modified in as far as regards the eruption, but proving fatal by affection of the brain.

William Timms, æt. 30, labourer in lead works, in the habit of frequent intoxication, was admitted a patient into the Small-pox Hospital, Sept. 21, 1820. He had been vaccinated by Mr. Griffin, surgeon, of Deddington, Oxfordshire, 18 years previously. Two vaccine cicatrices were very apparent on the left arm. The eruption proved to be distinct, and on the 7th day it was drying on the face, and exhibiting the usual tuberculous character of modified small-pox. Throughout the

- I may be permitted, perhaps, to remind those who have not been in the habit of seeing small-pox lately, that the eruption on the skin and throat is only one of the effects of the poison. Another, at least equally important, both with reference to pathology and practice, is that which is exerted upon the brain and nervous system; the chief evidences of which are delirium, inflamed eyes, stupor or restlessness, and disposition to erysipelas and gangrene. 
whole course of the disease, however, there were some obscure marks of affection of the brain. On the subsidence of the cutaneous inflammation, comatose symptoms came on, and he died on the following day. .

The brain, on dissection, exhibited no appearances of recent disease.

\section{CASE II.}

Variola succeeding vaccination, modified in as far as regards the eruption, but proving fatal by an $\otimes b$ scure affection of the brain.

Ruth Beddoes, æt. 19, of gross and plethoric habit of body, was admitted into the Small-pox Hospital, Sept. 28, 1822, with a distinct and mild eruption, attended, however, with considerable conjunctival inflammation. She had been vaccinated five years previously, at Bishop's Castle in Shropshire. The arm inflamed severely, and for a long time afterwards was kept in a sling. A very small vaccine cicatrix was observable in her left arm.

The cutaneous eruption passed through its stages rapidly; but the appetite never returned. On the eighth day, ophthalmia came on with great violence, yielding, however, to the free use of the lancet. Five days afterwards, the arm in which 
she had been bled became affected with severe erysipelas, which subsequently attacked the leg of the opposite side. Four days after this, violent delirium supervened, and she gradually sunk into a state of low typhus, in which she died. On opening the head, no morbid appearances of any kind could be detected, nor were there any traces of disease in the thorax or abdomen.

Having thus described the manner in which the effects of the variolous poison are modified by previons vaccination, I proceed to notice the degree to which such modification takes place. This varies very greatly; - to an extent, indeed, hardly conceivable by those who have not paid minute attention to the subject. Sometimes the disease, after vaccination, is so highly modified, that physicians can scarcely think themselves warranted in calling the complaint small-pox. It has all the characters of varicella, and has even been confounded with those slight papular eruptions which are met with in particular habits, and in irritable skins, more especially in warm weather.

On the other hand, the modification produced by previous vaccination is, in some few cases, so trifling, as hardly to be perceptible. Between these extremes, every possible gradation has been noticed; as the practice of the Small-pox Hospital, during the last year, has abundantly testified. 
It is highly satisfactory to know, that the ins stances of complete failure, from well-ascertained vaccination, are very few in number, and will hardly bear a comparison with those numerous instances, in which the disease was so modified by it, as to preclude all anxiety for the patient's safety. Of the fifty-seven cases of small-pox, after vaccination, admitted into the Small-pox Hospital in 1822, forty-four were discharged in perfect health within fourteen days from the period of their admission*. There were five fatal cases. The history of one of these (Ruth Beddoes) has been already detailed; and it is evident from the reported state of the arm, that the vaccination, in this instance, was not trustworthy. It is, moreover, fair to presume, from the high degree of inflammation which attended the process of vaccination, that there was a strong predisposition in this constitution to suffer from the variolous poison, and the result would, perhaps, have been equally fatal, had the patient taken small-pox by inoculation. The same degree of doubt attaches to the remaining four. In two of them, the cicatrices were very large and irregular. In one, no cicatrix was discernible; and in one only could it be said, that the scars were tolerably regular.

In the present state of the country, it appears highly desirable that some investigation should

* Table No.4 exhibits an abstract of the periods of their duration in hospital. 
take place into the real causes of the occurrence of small-pox after vaccination, in order to determine, if possible, how far there is any probability of our being able to obviate them in future. The following observations are thrown out with the view of assisting in the determination of this question :

1. Small-pox after vaccination, unquestionably prevails in particular families; showing, that in them there exists some peculiar susceptibility of the variolous poison. Various instances of the kind have fallen under my own immediate observation, the most striking of which is the following :

George Ferriman, æt. 30, was admitted into the Small-pox Hospital Oct. 28, 1822, with pretty severe modified small-pox; and with him were also admitted his two children, Thomas and Harriet, both labouring under a very slight form of the same disease. The father had been inoculated for small-pox when a child, and was always considered to have passed through the disease in a regular way. His children had been vaccinated, and their arms exhibited very perfect cicatrices.

I have seen small-pox attack three individuals of the same family, who had been vaccinated at different ages, in different places, and by different persons. I witnessed this, during the last summer, in the family of a medical practitioner, in the neighbourhood of Red Lion-square. 
2. It is certainly worthy of observation, that the great majority of cases of small-pox subsequent to vaccination, which have occurred at the Small-pox Hospital, have been persons between the ages of 15 and 21 . Nineteen is the average age of the whole, as the Table, No. 2, will show. How far this may depend upon accidental causes, or upon the length of time which has elapsed since the general diffusion of vaccination, I am unable to speak decisively. From the fact, however, that many of these persons had been frequently and thoroughly exposed to the contagion, at former periods of their lives, I am induced to entertain the notion, that there is something in the habit of body peculiar to that age, which renders the system more than usually disposed to suffer from the influence of the variolous poison.

The circumstance now adverted to has lately become obvious to the world, and it has revived an opinion entertained in the earlier days of vaccination, that its influence on the system wears out in the progress of life, and requires periodical renewals. The notion has latterly been acted upon to a great extent; but I have not been able to ascertain, that the results of revaccination correspond with the theory which leads to it.

3. In any investigation of the causes of smallpox subsequent to vaccination, it. would be improper to overlook the remarkable connection that 
subsists between the degree of perfection in the vaccine cicatrix, and the violence of the secondary disease. This important fact was forced upon my attention by the results of the last year's experience at the Small-pox Hospital. It is, indeed, in opposition to the opinion entertained by several authors of acknowledged reputation; but the extent of my opportunities enables me to speak with much confidence on this point. When the scar on the arm is perfect, - that is, distinct; circular, radiated, and cellulated; but, above all, when it is small, so that it may be covered by a pea;-the secondary affection (if from peculiarity of habit, or any other less ascertained cause, it does occur,) will be slight, and hardly deserve the name of a disease.

On the other hand, whenever the scar is large, and bears the marks of having been formed by high local inflammation, and wants the other distinctive characters just enumerated, the chance of small-pox occurring in after-life will be greater, and, cateris paribus, there will be a stronger likelihood of its proving severe.

This principle receives a striking confirmation from what takes place in revaccination. Where the cicatrix is perfect, it is impossible, or nearly so, to reproduce the vaccine disease in any thing like its genuine form. In proportion to the imperfection 
of the cicatrix, will be the degree of approximation of the second to the primary vaccination.

These considerations tend to establish, as a pathological principle, that the occurrence of smallpox, subsequent to vaccination, is dependent upon the intensity of the vaccine influence, as primarily exerted; and they lead to the belief, that the appearance of the cicatrix may be taken as a measure of that intensity.

4. The last point to which I am desirous of directing the attention of the Society is the fact, that a very large proportion of those persons who have been admitted, during the last three years, into the Small-pox Hospital, having the disease subsequent to vaccination, had been vaccinated in the country. This may be considered, perhaps, as an accidental circumstance. It may be argued, that the domestic servants in London, who, of all persons, may most naturally be expected to resort to an hospital in the time of sickness, are, in a great measure, supplied from the country. It may possibly be owing, in part, to the susceptibility of the disease being increased (here, as in other instances) by change of air; but, making all necessary allowances, the disproportion between those who take small-pox, after vaccination in the country, and after vaccination in London or some other large town, still appears to me so great, that we must seek for an explanation which may have a 
wider range of influence. I have long been impressed with the notion, that practitioners in the country have frequently vaccinated with lymph, which was not perfect in its qualities. I am inclined to entertain this opinion, first, from having been able to trace several cases of small-pox after vaccination to particuldr villages, in counties bordering on the metropolis; and, secondly, from having observed, that a great proportion of those admitted into the Small-pox Hospital after country vaccination, had large, irregular; and therefore $i m$. perfect, cicatrices.

If the notions which I entertain of the influence of vaccination on the animal economy, and on the causes of its occasional failure, are correct, their general diffusion might assist, not only in upholding vaccination as an object of great national and individual importance, but in checking those unpleasant occurrences which are now making such alarming inroads on the confidence of the public in the vaccine protection. It would induce practitioners to revaccinate, with fresh and genuine lymph, those whose arms do not exhibit the perfect cicatrix ; -it would lead those in the country to apply more frequently to large towns for a supply of recent lymph;-it would point out the propriety of their putting less confidence than heretofore in points and preserved lymph;-and it might impress upon all the indispensable necessity of a close attention to every part of that process, 
which, though of trifling import at the moment, is yet of incalculable consequence to individuals in every future period of their lives.

\section{A P P E N D I X.}

$$
\text { No. } I \text {. }
$$

List of patients having small-pox after vaccination, admitted into the Small-pox Hospital, in two different periods, of five years each; showing the proportion such cases bear to the whole number of admissions.

\begin{tabular}{|c|c|c|c|}
\hline Year. & $\begin{array}{l}\text { Total } \\
\text { admis- } \\
\text { sions. }\end{array}$ & $\begin{array}{c}\text { Total } \\
\text { of } \\
\text { Small- } \\
\text { pox } \\
\text { after } \\
\text { Vacci- } \\
\text { nation. }\end{array}$ & $\begin{array}{l}\text { Proportion of } \\
\text { Cases of Small- } \\
\text { pox after Vacci- } \\
\text { nation, to the } \\
\text { whole number } \\
\text { of admissions. }\end{array}$ \\
\hline 1809 & 146 & 4 & One in 36 \\
\hline 1810 & 149 & 5 & 30 \\
\hline 1811 & 94 & 6 & 15 \\
\hline 1814 & 79 & 4 & 20 \\
\hline 1815 & 101 & 6 & 17 \\
\hline $\begin{array}{c}\text { Total in } 5 \\
\text { years. }\end{array}$ & 569 & 25 & 22 \\
\hline 1818 & 58 & 9 & 6 \\
\hline $\begin{array}{c}1819 \\
\text { To Sept.7. }\end{array}$ & 97 & 17 & 6 \\
\hline 1820 & 142 & 25 & 6 \\
\hline 1821 & 117 & 28 & 4 \\
\hline 1822 & 194 & 57 & $3 \frac{1}{2}$ \\
\hline $\begin{array}{c}\text { Total in } 5 \\
\text { years. }\end{array}$ & 608 & 136 & $4 \frac{1}{2}$ \\
\hline
\end{tabular}




\section{No. $I I$.}

Table of the ages of the different patients affected with small-pox after Vaccination, who have been admitted into the Small-pox Hospital during the last five years.

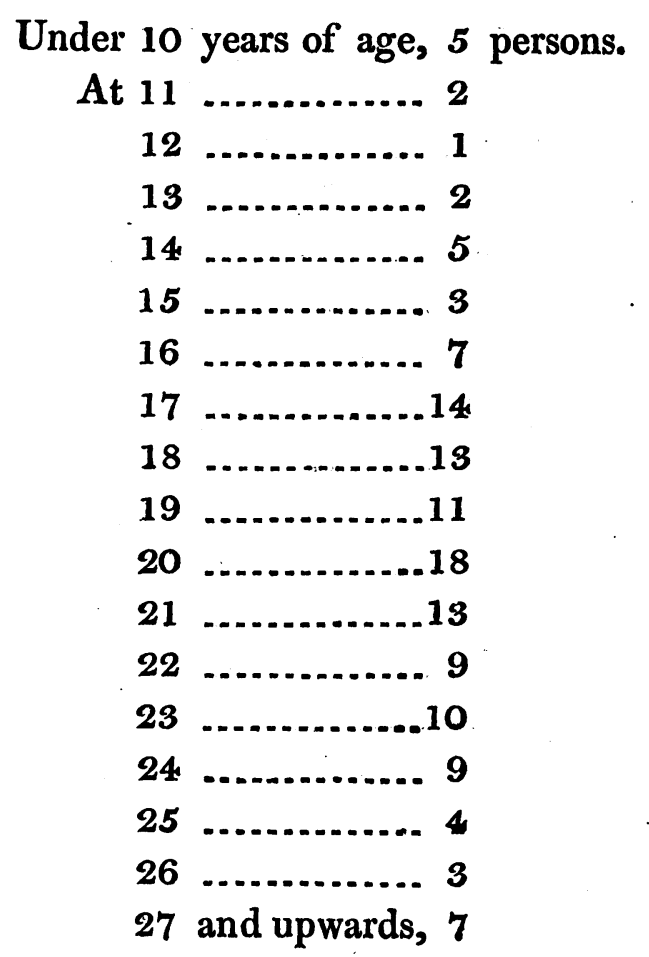

136 


\section{No III.}

List of patients having Small-pox after Vaceination; distinguishing those vaccinated in the country from those vaccinated in toron, and those not ascer. tained.

\begin{tabular}{|c|r|r|r|r|}
\hline & \multicolumn{2}{|c|}{ Vaccinated. } \\
\cline { 2 - 4 } Year. & $\begin{array}{c}\text { In the } \\
\text { coun- } \\
\text { try. }\end{array}$ & $\begin{array}{c}\text { In } \\
\text { Lon- } \\
\text { don. }\end{array}$ & $\begin{array}{c}\text { Not as- } \\
\text { tained } \\
\text { there. } \\
\text { wacci- } \\
\text { nated. }\end{array}$ & \\
\hline 1809 & 9 & 1 & 0 & 4 \\
1810 & 4 & 0 & 1 & 5 \\
1811 & 5 & 0 & 1 & 6 \\
1814 & 4 & 0 & 0 & 4 \\
1815 & 5 & 1 & 0 & 6 \\
1818 & 7 & 0 & 2 & 9 \\
1819 & 11 & 1 & 5 & 17 \\
To Sept.7. & & & & \\
1820 & 6 & 1 & 18 & 25 \\
1821 & 19 & 2 & 7 & 28 \\
1822 & 35 & 6 & 16 & 57 \\
\hline Total. & 99 & 12 & $50 *$ & 161 \\
\hline
\end{tabular}

* There is every probability, that the fifty persons set down in this column, were vaccinated in the country; -as residents in town would hardly have expressed any doubts on the subject. 


\section{No. IV.}

Table, exhibiting the duration in hospital, of the cases of small-pox after vaccination, admitted into the Small-pox Hospital during the year 1822.

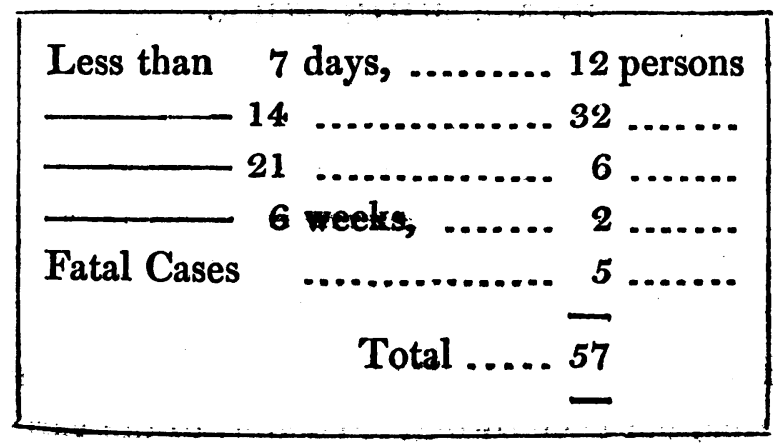

